\title{
Zur Einfïhrung.
}

Die experimentelle Forschung ist für die Entwicklung der Medizin von Jahr zu Jahr bedeutungsvoller geworden. Für die theoretischen Fächer wird das Experiment längst als unentbehrlich angesehen, und auch viele Fortschritte in der praktischen Medizin beruhen auf Ergebnissen experimenteller Studien.

Fruchtbringende klinische und theoretische Arbeit hat darum in viel höherem Maße als früher die Kenntnis einschlägiger experimenteller Untersuchungen zur Voraussetzung. Für die experimentelle Forschung selbst besteht andrerseits die Gefahr der Zersplitterung, weil die Einzelergebnisse nicht immer unter großen Gesichtspunkten zusammengefaßt werden. Hieran ist vor allen Dingen die Tatsache schuld, daß die Arbeiten klinischer und theoretischer Forscher bisher in verschiedenen Zeitschriften niedergelegt wurden. So kam es, daß der wechselseitige Einfluß zwischen theoretischen und praktischen Disziplinen häufig zurücktrat, der sonst eine Fülle von Anregung und Befruchtung geboten hätte.

Die experimentelle Forschung kann erst dann zu ihrer vollen Bedeutung gelangen, wenn ihre Einzelrichtungen sich nach Möglichkeit zusammenschließen und in engster Verbindung miteinander arbeiten.

Das Bedürfnis, die Ergebnisse experimenteller Arbeit zu vereinigen und só allen Teilen zugute kommen zu lassen, ist unverkennbar geworden. Es findet seine Parallele in den Bestrebungen, große Gebiete der Medizin überhaupt zusammenschließend zu behandeln. Darum haben sich die Herausgeber der neuen Zeitschrift zusammengefunden, um in ähnlicher Weise, wie es im Ausland bereits geschah, auch im deutschen Sprachgebiet eine Sammelstätte für Arbeiten aus dem Gesamtgebiete der experimentellen Medizin zu begründen.

Bei der Auswahl der Arbeiten für unsere Zeitschrift wird nach den gekennzeichneten Gesichtspunkten verfahren. Nur Arbeiten aus dem Gebiet der experimentellen Medizin, die einen Fortschritt bedeuten und allgemein medizinisches Interesse haben, sollen berïcksichtigt werden.

Es folgt daraus, daß wir für eng begrenzte Spezialuntersuchungen keinen Raum haben. Andererseits wird aber den Wünschen der Autoren 
und den Bedürfnissen der Leser dadurch besonders gedient, da $\bar{B}$ alle angenommenen Arbeiten sofort in Druck gelegt werden und spätestens innerhalb 6 Wochen erscheinen.

Die Gründung einer Zeitschrift für die gesamte experimentelle $\mathrm{Me}$ dizin war von einem Teil der Herausgeber seit langer Zeit beabsichtigt. Das Unternehmen wird nach unserer Uberzeuğung bald der lebhaften Beteiligung und Förderung seitens der wissenschaftlich arbeitenden Kreise sich erfreuen.

E. Abderhalden. E. Enderlen. B. Krönig. C. v. Noorden. E. Payr. C. v. Pirquet. F. Sauerbruch. A. Schittenhelm. W. Straub. W. Trendelenburg. P. Uhlenhuth. 